\title{
Characterization of site-specific glycosylation of secreted proteins associated with multi-drug resistance of gastric cancer
}

\author{
Jian Wu ${ }^{1, *}$, Hongqiang Qin ${ }^{2,}{ }^{,}$, Ting $\mathrm{Li}^{1}$, Kai Cheng ${ }^{2}$, Jiaqiang Dong ${ }^{1}$, Miaomiao Tian ${ }^{1}$, \\ $\mathrm{Na}$ Chai ${ }^{1}$, Hao Guo ${ }^{1}$, Jinjing $\mathrm{Li}^{1}$, Xin You ${ }^{2}$, Mingming Dong ${ }^{2}$, Mingliang $\mathrm{Ye}^{2}$, Yongzhan \\ Nie $^{1}$, Hanfa Zou ${ }^{2}$, Daiming Fan ${ }^{1}$ \\ ${ }^{1}$ State Key Laboratory of Cancer Biology and Xijing Hospital of Digestive Diseases, Fourth Military Medical University, Xi'an \\ 710032, China \\ ${ }^{2}$ Key Laboratory of Separation Sciences for Analytical Chemistry, National Chromatographic R \& A Center, Dalian Institute of \\ Chemical Physics, Chinese Academy of Sciences, Dalian 116023, China \\ *These authors contributed equally to this work
}

Correspondence to: Daiming e-mail: Fan, daimingfan@fmmu.edu.cn Hanfa Zou, e-mail: hanfazou@dicp.ac.cn

Keywords: gastric cancer, secretome analysis, chemoresistance, glycoprotein, site-specific glycosylation

Received: October 14, $2015 \quad$ Accepted: March 06, 2016

Published: March 23, 2016

\section{ABSTRACT}

Multi-drug resistance (MDR) remains a great obstacle to effective chemotherapy for gastric cancer. A number of secreted glycoproteins have been reported to be involved in the development of MDR in gastric cancer. However, whether glycosylation of secreted glycoproteins changes during MDR of gastric cancer is unclear. Our present work manifested that $\mathbf{N}$-glycosites and site-specific glycoforms of secreted proteins in drug-resistant cell lines were distinctly different from those in the parental cell line for the first time. Further characterization highlighted the significance of some aberrantly glycosylated secretory proteins in MDR, suggesting that manipulating the glycosylation of specific glycoproteins could be a potential target for overcoming multi-drug resistance in gastric cancer.

\section{INTRODUCTION}

Gastric cancer (GC) ranks the second most commonly diagnosed malignancy and the second most lethal cancer in China [1]. Despite the great improvements in diagnostic and therapeutic techniques of gastric cancer, the 5-year overall survival of GC patients still remains unsatisfactory. And, the emergence of multi-drug resistance (MDR) is largely responsible for the dismal long term survival of GC patients [2].

To date, there have been extensive studies on the mechanism of MDR. And, a number of molecules such as ATP-binding cassette (ABC) transporters have been found to be involved in the development of MDR [3]. To explore the underlying mechanisms of MDR in GC, the multidrug resistant cell lines, SGC7901/ADR and SGC7901/ VCR, were derived from human gastric adenocarcinoma cell line SGC7901 by stepwise selection with adriamycin and vincristine respectively in our lab. Based on these drug-resistant cell models, we have discovered a series of microRNAs (miR-19a/b, miR-508-5p, miR-27b, miR129-5p) and proteins (HIF-1 $\alpha$, MGr1-Ag/37LRP, CCNG1) that could modulate the development of MDR in gastric cancer [4-8]. Besides, a growing number of researches concerning the role of tumor microenvironment in MDR declare that macrophages, $\mathrm{T}$ cells, fibroblasts and ECM (extracellular matrix) within tumor microenvironment could contribute to MDR of multiple cancers by direct and indirect interactions with tumor cells [9-12]. As critical components of the tumor microenvironment, secreted proteins of tumor cells also have been demonstrated to be greatly involved in chemoresistance. Targeted therapy with BRAF, ALK or EGFR kinase inhibitors induces a complex network of secreted factors in human melanoma and lung adenocarcinoma cells, and this therapy-induced secretome stimulates the outgrowth and dissemination of drugresistant cancer cells and promotes the survival of drugsensitive cancer cells [13]. Besides, extracellular vesicles derived from drug-resistant cancer cells could confer drug resistance on drug-sensitive cancer cells $[14,15]$. Secreted clusterin (sCLU) was reported to contribute to MDR of several cancers such as osteosarcoma and hepatocellular carcinoma $[16,17]$. In another example, secreted sonic hedgehog was proved to be of great significance in the 
development of MDR in multiple myeloma [18]. As for gastric cancer, Yuan et al. claimed that WNT6, a secreted glycoprotein, promoted multi-drug resistance of gastric cancer [19], and Cho et al. indicated that VEGF-C secretion contributed to chemoresistance of gastric cancer to cisplatin [20]. More importantly, the proteomic research accomplished by Huang et al. reported that secreted proteins were quite different between drug-sensitive and drug-resistant gastric cancer cell lines [21].

As one of the most abundant post-translational modifications (PTMs), glycosylation exercises great influences on not only structures but also functions of glycoproteins. Our previous study demonstrated that P-gp, a pivotal player in MDR, was aberrantly glycosylated in MDR gastric cancer cell lines and glycosylation at Asn99 residue might greatly affect its function in MDR [22]. Intriguingly, recent researches indicated that glycomic alterations of glycoproteins exerted enormous effects on chemoresistance of cancers. Lattova et al. verified the correlations between $\mathrm{N}$-glycosylation variations and the efficacy of chemotherapies in breast cancer cells [23, 24] and discovered that the emergence of galactosylated or biantennary fucosylated glycans might be associated with chemoresistance. Besides, Nakano et al. observed that a 2-6 sialylation of $\mathrm{N}$-glycans decreased with acquired resistance to desoxyepothilone B in leukemia cells [25]. Yet, the former researches mainly focused on glycosites or glycan structures. But, there are many glycosites in one glycoprotein, as well as many glycans at the same glycosite, which makes the glycosylation much more complex. Intact glycopeptides simultaneously contain the sequence of peptides, glycosites as well as glycan structures corresponding to the sites. And there have been several reports about the determination of sitespecific glycoforms by MS, including $\mathrm{N}$ - and O-linked glycosylation [26-28]. In our previous work, site-specific glycoforms of total cell proteins were determined by combining de-glycopeptide and intact glycopeptide analysis [29]. Up to now, however, the identification and quantification of intact glycopeptides on large-scale remains a huge challenge, especially for the analysis of glycosylation of low abundance secreted glycoproteins.

Herein, we fabricated a platform for characterizing the glycosylation heterogeneity of secreted proteins in our MDR models, which included concentration of secreted proteins, enrichment of intact glycopeptides and determination of site-specific glycoforms. Additionally, the glycosites and site-specific glycoforms of secreted proteins were quantified, which comprised glycosite occupancy in protein as well as different glycan structures on the same glycosite. Totally, 1033 glycosites were identified from secreted proteins of SGC7901, SGC7901/ ADR and SGC7901/VCR, including 240 sites with different glycosylation occupancy. A total of 2222 sitespecific glycoforms were determined, and 499 site-specific glycoforms on 151 sites were significantly different between SGC7901 and its MDR sublines. To the best of our knowledge, this is the first large-scale analysis of sitespecific glycoforms of secreted proteins in gastric cancer. Consequently, the comprehensive characterization of sitespecific glycosylation could help us better understand the sophisticated mechanism of MDR in gastric cancer, thus promoting the discovery of feasible biomarkers for MDR and improving the efficiency of chemotherapy.

\section{RESULTS}

\section{Generation of secretome datasets from 3 gastric cancer cell lines}

As stated above, the secretome of tumor cells played important roles in the process of acquired chemoresistance. Consequently, the analysis of secreted glycoproteins on proteome level could help us understand the specific mechanism of drug resistance. Due to the very low abundance, the analysis of secreted glycoproteins is still a big challenge, especially for gastric cancer cells and tissues. Thus, the secreted proteins need to be concentrated before analysis. As shown in Figure 1, the serum-free culture medium was collected and then concentrated by ultra filters (UF). The secreted proteins with low abundance could be effectively concentrated, and the salts in medium could be removed at the same time. Additionally, the glycopeptides were enriched by using hydrophilic interaction liquid chromatography (HILIC), which could retain the glycan structures linked to glycosites with high specificity. For the determination of site-specific glycoforms, the information of backbone peptide sequence, glycosite location and glycan structures was obtained by analysis of de-glycopeptides and intact glycopeptides, respectively.

Generally speaking, a total of 1033 N-linked glycosites (localization probability $>0.75$ ), mapping to 436 non-redundant $\mathrm{N}$-glycoproteins (shown in Supplementary Table S1), were quantified from secreted proteins of the three cell lines (SGC7901, SGC7901/ADR and SGC7901/VCR), among which $613 \mathrm{~N}$-glycosites and $286 \mathrm{~N}$-glycoproteins were quantified in all the three cell lines. In addition, we totally identified $2222 \mathrm{~N}$-linked sitespecific glycoforms with high confidence. The detailed information of N-linked glycosites, $\mathrm{N}$-glycoproteins and site-specific glycoforms identified from each cell line is summarized in Figure 2.

To verify whether the identified glycoproteins were secretory proteins, we applied bioinformatics softwares and Uniprot database to predict protein secretion pathway. 272 proteins were predicted to be secreted in the classical secretory pathway (138 proteins predicted by SignalP program and 134 proteins including the Uniprot keyword "secreted and/or signal"). In addition, the SecretomeP software presented that 74 proteins were secreted through the nonclassical secretory pathway. In short, these results 
manifested that $79 \%$ of the identified glycoproteins could be released into the conditioned medium by different secretory pathways, demonstrating high efficiency of the platform for analysis of secreted protein glycosylation (Supplementary Figure S1).

To further characterize the identified secreted glycoproteins, protein ontology analysis was executed by Panther software to interpret the functions and involved pathways of the 346 secreted glycoproteins. As shown in Supplementary Figure S2, these glycoproteins had multiple functions and participated in diverse pathways that might play vital roles in the development of MDR. The top three most common molecular functions were catalytic activity $(32.8 \%)$, binding $(27.0 \%)$ and receptor activity $(25.0 \%)$. Besides, the major involved pathways related to MDR were integrin signaling pathway, TGF- $\beta$ signaling pathway, cadherin signaling pathway, angiogenesis, Wnt signaling pathway and apoptosis signaling pathway.
Then we, for the first time, attempted to illuminate the possible correlations of site-specific glycosylation of glycoproteins with MDR through comprehensively analyzing the changes of glycosites and site-specific glycoforms of secreted proteins during MDR in gastric cancer.

\section{Characterization of N-glycosite occupancy varying with MDR}

Because of significant changes in glycosite occupancy during MDR, the quantification of glycosites was important for studying MDR. We compared the $\mathrm{N}$-glycosites of SGC7901 cell line with those of two drug-resistant cell lines, respectively. About 81 glycosites were only quantified in both of the MDR cell lines, while 75 glycosites were only identified in secreted proteins of SGC7901 (Figure 2A, Supplementary Table S1). Additionally, among the 613 glycosites identified in all the

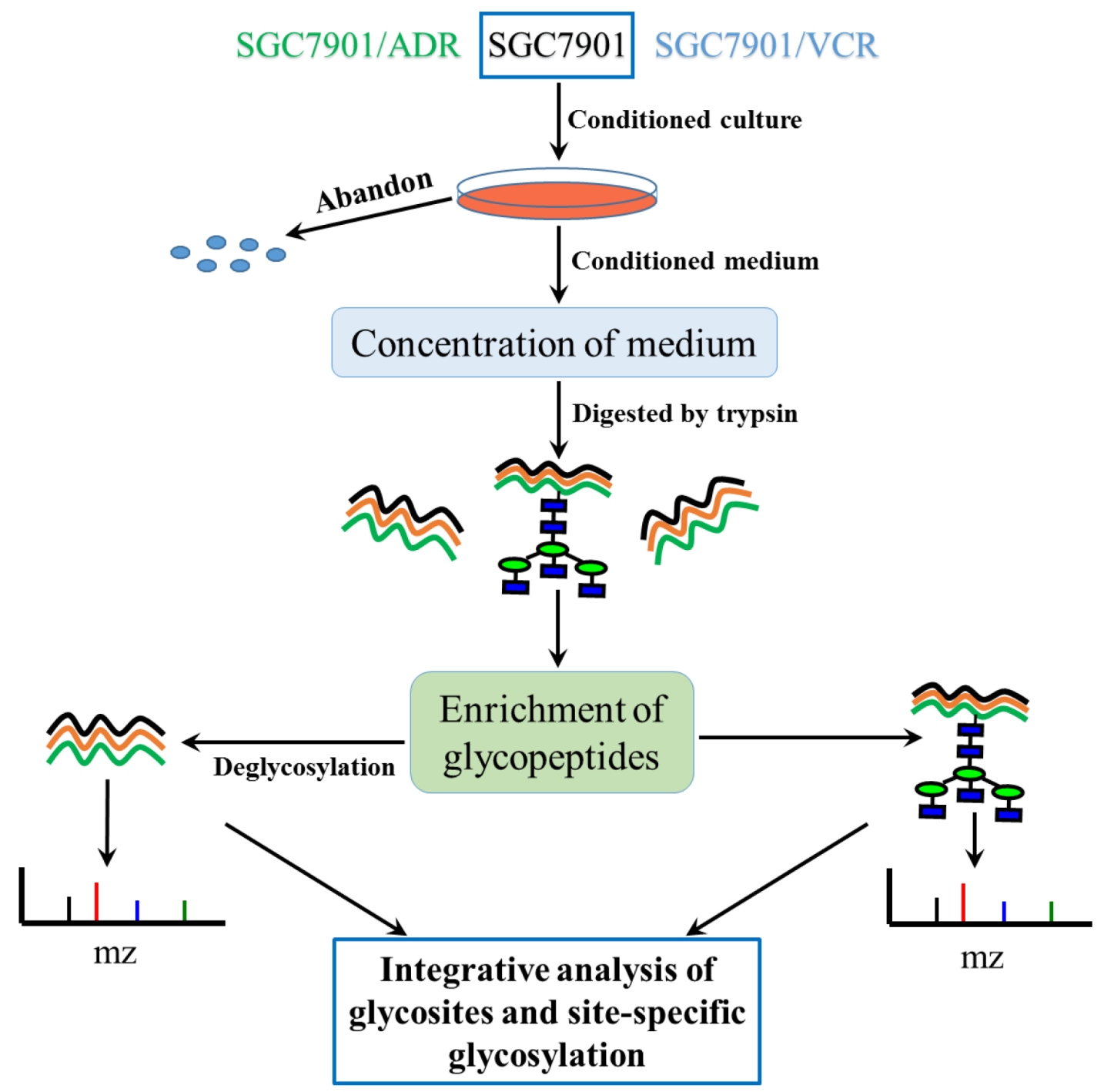

Figure 1: Workflow for the characterization of site-specific glycosylation of glycoproteins in secretomes of SGC7901, SGC7901/ADR and SGC7901/VCR. 
three lines, 84 glycosites in secreted glycoproteins were significantly changed between SGC7901 and the MDR cell lines with $p$-value $\leq 0.01$ by $t$-test (Figure 3 ). Totally, $240 \mathrm{~N}$-glycosites were found dramatically changed in SGC7901-ADR/VCR (124 increased and 116 decreased) compared to SGC7901, as illustrated in Supplementary Table S1. And the significantly different glycosites could be mapped to 163 glycoproteins ( 84 glycoproteins for upregulated glycosites, 76 glycoproteins for down-regulated glycosites). Interestingly, there are 3 proteins (IGF2R, ITGB1 and PTPRF) that both have up-regulated and down-regulated glycosites, among which 2 glycoproteins (IGF2R, ITGB1) have been demonstrated to be involved in drug-resistance. Interestingly, as a major player in chemoresistance, ITGB1 (integrin beta-1) could mediate the anti-apoptosis effects of TIMP1 (further discussed below) in melanoma [30].

Further bioinformatics analysis indicated that the leading three molecular functions of 87 glycoproteins for increased glycosites were binding $(32.4 \%)$, receptor activity $(25.4 \%)$ and catalytic activity $(23.9 \%)$, while for 79 glycoproteins corresponding to decreased glycosites, catalytic activity (33.3\%), receptor activity (25.0\%) and binding $(25.0 \%)$ ranked the top three. And these three molecular functions were closely related to drug resistance. Moreover, in terms of specific pathways, the numbers of annotated glycoproteins in category of increased glycosites outperformed those in category of decreased glycosites. For example, 12 glycoproteins in Integrin signaling pathway and 4 glycoproteins in TGFbeta signaling pathway were annotated in the cluster of glycoproteins with increased glycosites, much more than those annotated in the cluster of glycoproteins for decreased glycosites. The detailed information was provided in Figure 4. It seemingly highlighted the importance of increased glycosites and their corresponding glycoproteins in the rise of MDR.

\section{Identification of altered site-specific glycoforms between SGC7901 and its MDR counterparts}

Previous studies had demonstrated that N-glycan profiles changed remarkably after chemotherapy in

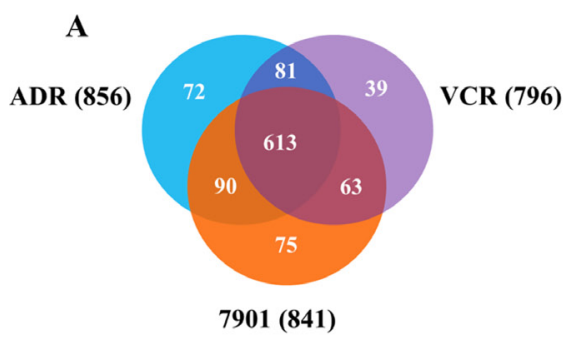

N-linked Glycosites $(\mathbf{1 0 3 3}$, local prob $>0.75)$

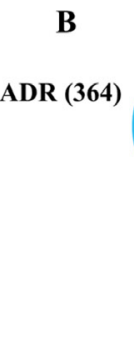

N-linked Glycoproteins (436) various cancers, and multiple $\mathrm{N}$-glycomic alterations were prominently associated with drug resistance [22, 31]. Ma et al. [31] reported that increased core-fucosylated and sialylated glycans were, to some extent, responsible for MDR in breast cancer cells. However, it was not the same case in MDR of gastric cancer. In the present study, we merely found increased core-fucosylation and hybrid glycans after acquired drug resistance in gastric cancer cell lines, while the other major glycoforms did not show distinct changes. More interestingly, the two MDR sublines exhibited diverse glycomic changes compared to their parental cell line in most cases (Supplementary Table S2). This might be caused by the heterogeneity of glycan structures onto the glycosites. Nevertheless, we maintained that this result could not fully represent the comprehensive glycomic alterations during MDR, as glycosylation contained the information of peptide backbone sequences, glycosite location and glycan structures, simultaneously. Thus, the determination of sitespecific glycoforms in secreted proteins could completely reveal the heterogeneity of glycosylation. As shown in Figure $2 \mathrm{C}$, about $31 \%(678 / 2222)$ of the glycoforms were identified in at least two cell lines and the overlap of sitespecific glycoforms of three gastric cancer cell lines was merely $12 \%(272 / 2222)$ of the total identified glycoforms, which demonstrated that site-specific glycoforms did dramatically change between drug sensitive and resistant cancer cells. The detailed information of sitespecific glycoforms of each cell line was displayed in Supplementary Table S2.

Moreover, we further characterized the significantly different site-specific glycoforms between SGC7901 and its MDR counterparts. As shown in Supplementary Table S2, 175 site-specific glycoforms were increased in secreted proteins of MDR cell lines, while 324 glycoforms were decreased. Interestingly, we discovered that some glycosites contained both up-regulated and downregulated glycoforms, indicating the high complexity of glycosylation. Totally, 499 site-specific glycoforms onto 151 glycosites in 106 glycoproteins were significantly changed between SGC7901 and its MDR derivates. Further analysis of the mapped glycoproteins showed that most of the annotated glycoproteins were involved in pathways

\footnotetext{
Figure 2: Venn diagram for numbers of N-glycosites, N-glycoproteins and site-specific glycoforms identified in SGC7901 and its MDR derivates. 7901: SGC7901; ADR: SGC7901/ADR; VCR: SGC7901/VCR.
} 
of Integrin, P53, Wnt, Notch, TGF-beta and Hedgehog (Supplementary Figure S3), all of which had been reported to play critical roles in drug resistance of cancer.

\section{Integrative characterization of changed sites and altered site-specific glycoforms}

As described above, both of the glycosites and sitespecific glycoforms were greatly different between drugsensitive and drug-resistant cell lines. Notably, we found that the glycosites with significant changes on glycositelevel rarely intersected with those corresponding to altered site-specific glycoforms (Supplementary Table S3). Moreover, we analyzed the glycosites with significantly altered site-specific glycoforms, and found that about $54 \%$ of the glycosites contained more than two significant glycoforms (shown in Supplementary Figure S4), which indicated the high heterogeneity of glycosylation during MDR process. And a total number of 57 glycoproteins with more than 2 significantly different glycoforms onto
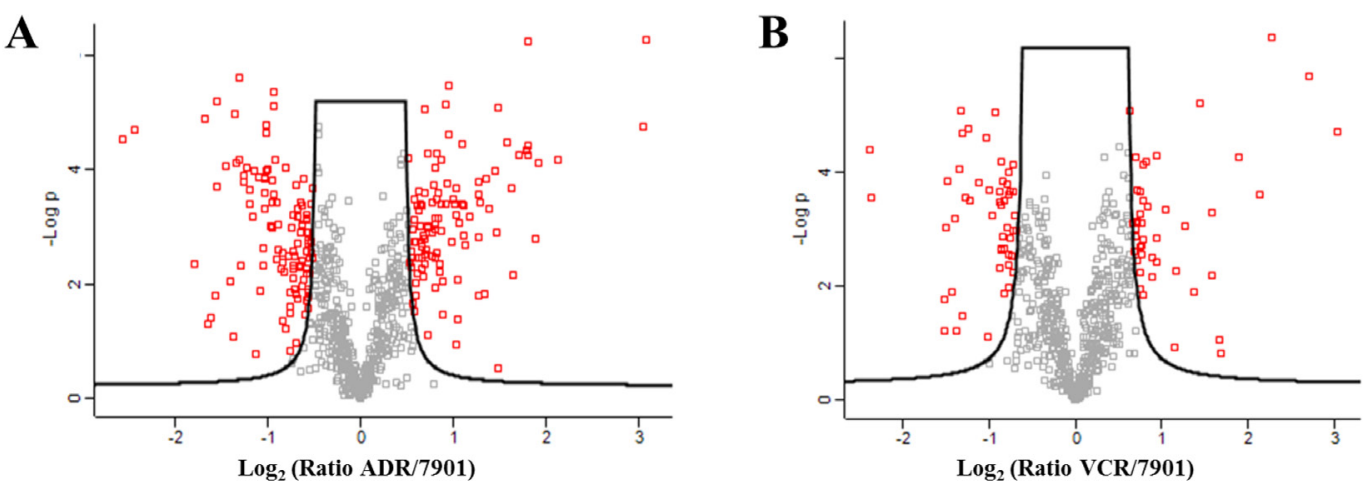

Figure 3: The comparison of glycosites identified in SGC7901 with those identified in two MDR sublines respectively. 7901: SGC7901; ADR: SGC7901/ADR; VCR: SGC7901/VCR; and the differentially expressed glycosites with $p$-value $\leq 0.01$ are marked red.

A

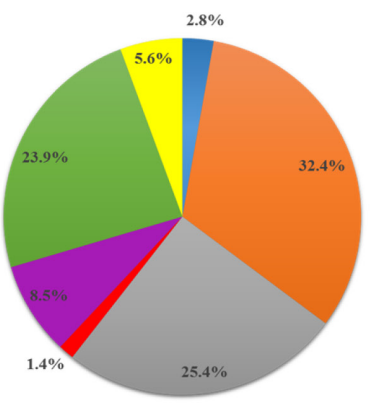

m translation regulator activity

a binding

meceptor activity

- enzyme regulator activity

- structural molecule activity

watalytic activity

transporter activity

C Molecular function

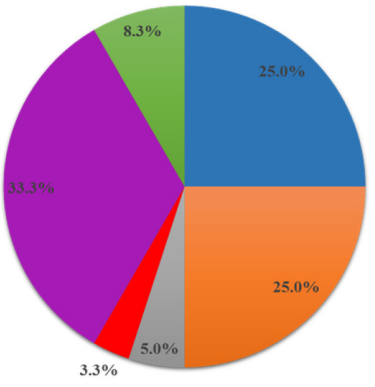

- binding

- receptor activity

= enzyme regulator activity

- structural molecule activity

- catalytic activity

- transporter activity

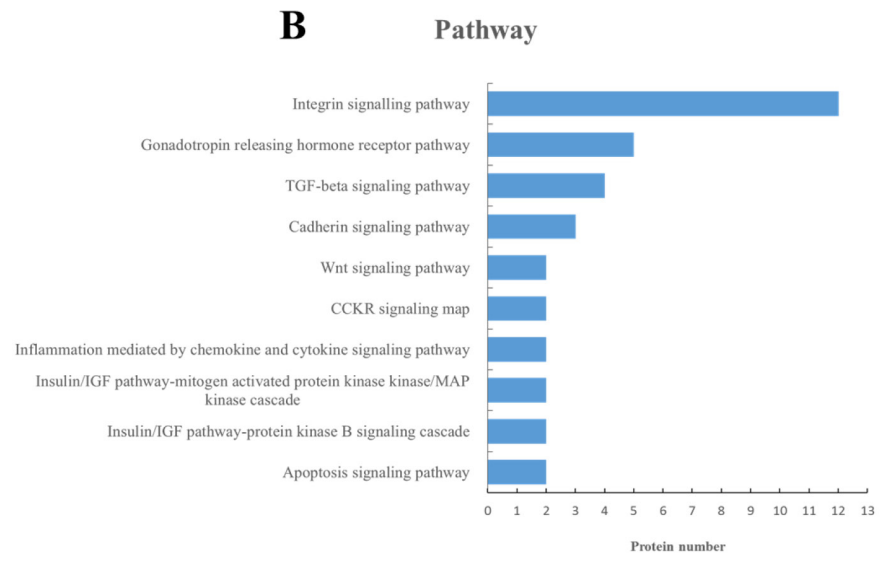

D Pathway

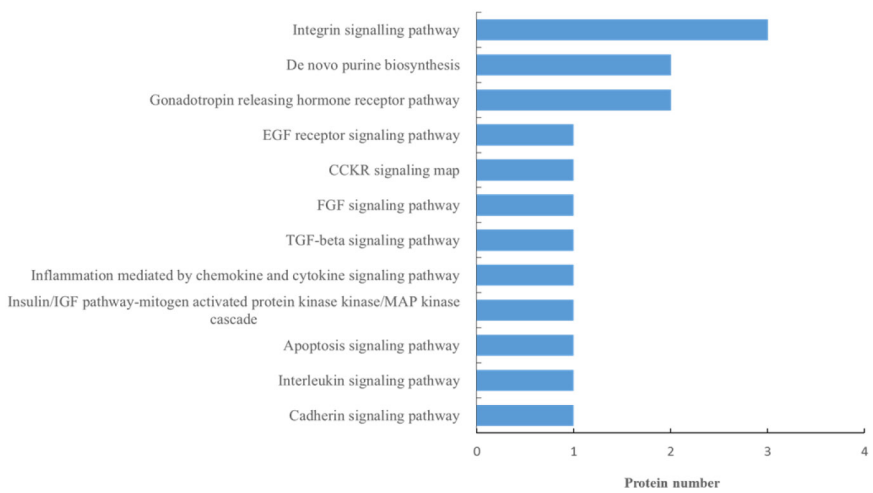

Figure 4: Ontology analysis of 163 glycoproteins corresponding to significantly different glycosites. Categories of molecular functions and involved pathways of glycoproteins for increased glycosites (A, B) and glycoproteins for decreased glycosites $(\mathbf{C}, \mathbf{D})$ in drug-resistant cells. 
one specific glycosite were identified (Supplementary Table S4). 33 of the glycoproteins had been reported to be associated with MDR of multiple cancers by searching in PubMed (Table 1). Thus, the quantification of glycosites combined with determination of glycoforms could preferably demonstrate the relationship between changes of glycosylation and MDR. The identified glycoproteins with significant changes on site-level and glycoformlevel would be the key points of our further research on chemoresistance in gastric cancer.

\section{DISCUSSION}

In the current study, we provided the most comprehensive secretome glycoproteomic characterization of SGC7901 and its MDR counterparts SGC7901/ADR, SGC7901/VCR. These three gastric cancer cell lines are extensively and intensively studied in our lab to elucidate the underlying mechanism of MDR in GC using genomic and proteomic approaches [32,33]. However, these studies mainly focused on the protein expression, but provided little information about protein glycosylation.

Herein, for the first time, we explored the correlations between glycosylation, particularly sitespecific glycosylation, in secreted glycoproteins and multidrug resistance in gastric cancer. Totally, 1033 $\mathrm{N}$-glycosites in 436 glycoproteins and $2222 \mathrm{~N}$-linked sitespecific glycoforms were identified and quantified from secreted proteins of SGC7901 and its MDR counterparts. It was noteworthy that core-fucosylation was increased in MDR cells. And, others had reported similar results in breast cancer cells [31] and prostate cancer cells [28]. Moreover, overexpression of two fucosyltransferases (FUT8 and FUT11) was probably responsible for the change of fucosylation observed in MDR cells [28]. Cheng et al. demonstrated that the alterations of FUTs (FUT4, FUT6 and FUT8) were involved in MDR in human hepatocellular carcinoma cells by modulating PI3K/Akt signaling pathway and MRP1 expression [34]. In our study, the increased fucosylation may be due to the up-regulation of FUT8 and FUT11 based on our mRNA sequencing results (data not shown). But, this hypothesis needs to be validated by further studies.

Besides, our preliminary results manifested that glycosites and site-specific glycoforms of secreted glycoproteins in MDR cells vastly differed from those in the parental ones. Compared to SGC7901, about 499 site-specific glycoforms in 106 glycoproteins were significantly changed with the similar trend in the two MDR cell lines. We further identified 57 glycoproteins with more than 2 significantly different site-specific glycoforms at one specific glycosite between drugsensitive and resistant cell lines, and found that more than half of them had been demonstrated to be involved in chemoresistance of various cancer types, among which the key roles of AXL, L1CAM, TIMP1 and Clusterin in
MDR were massively studied. Yet, there are few reports about the relationship between glycosylation of secretory proteins and MDR.

Here, we would comprehensively discuss the changes of glycosylation of four candidate glycoproteins during MDR by integrative analysis of glycosites and sitespecific glycoforms. The receptor tyrosine kinase AXL is over-expressed in different types of cancers and implicated in several malignant phenotypes of tumor cells, such as invasion and chemoresistance $[35,36]$. AXL could mediate multi-drug resistance by modulation of microRNAs and EMT (epithelial-to-mesenchymal transition), as well as signaling pathways of PI3K/Akt and EGFR/PKC/mTOR [37-41]. More importantly, aberrant glycosylation of AXL was reported to contribute to tumor proliferation, invasion and metastasis [42], and alpha-2, 6-sialyltransferases ST6GalNAcII was further identified as critical modulator of its functions in mammary phyllodes tumors [43]. In this work, we characterized the changes of site-specific glycosylation in secretory AXL during the acquisition of MDR in stomach cancer for the first time. Generally speaking, we identified three N-glycosites - Asn198, Asn339 and Asn345, among which Asn198 was found significantly reduced in MDR cells. Intriguingly, seven significantly differentially expressed glycoforms were quantified at Asn198 and the other two glycosites were identified without distinctly altered $\mathrm{N}$-glycans (Figure 5A, Supplementary Table S5), which possibly implied the importance of Asn198 in the actions of AXL. Soluble AXL may result from the proteolytic cleavage of receptor tyrosine kinase AXL [44]. It was reported that soluble AXL could outperform AFP in diagnosing very early hepatocellular carcinoma [45] and correlated with tumor stage as well as patient survival in renal cell carcinoma [46]. While, our further work validated the increased mRNA expression of AXL in GC MDR cells and increased protein expression of soluble AXL in the conditioned medium of GC MDR cells. In addition, we found that high expression of tumor AXL predicted worse survival in GC patients based on TCGA database (Figure 6). In a word, glycosylation and expression differences of soluble AXL between GC drug-sensitive and resistant cells have been observed, suggesting a significant role of soluble AXL in the MDR of GC, but this hypothesis requires further researches to explore how soluble AXL and aberrantly glycosylated AXL influence the MDR of GC.

We also characterized the site-specific glycosylation changes of three other proteins-L1CAM, TIMP1 and CLU, during MDR of GC cells (Figure 5B-5D, Supplementary Tables S6-S8). These proteins are all heavily glycosylated proteins and known to be involved in resistance to chemotherapies in multiple cancer types [47-52] [16, 53, 54]. Moreover, as glycoproteins, the functions of TIMP1 and CLU were reported to be markedly influenced by altered glycans [55-57]. Nevertheless, previous studies rarely focused on the 
Table 1: 33 MDR-related glycoproteins with significantly altered glycoforms at one specific site $\geq 2$

\section{Uniprot accession}

number
Protein name
Gene name

\begin{tabular}{|c|c|c|}
\hline P01033 & Tissue inhibitor of metalloproteinase 1 & TIMP1 \\
\hline P07602 & Proactivator polypeptide & PSAP \\
\hline $\mathrm{P} 11047$ & Laminin subunit gamma-1 & LAMC1 \\
\hline P32004 & Neural cell adhesion molecule L1 & L1CAM \\
\hline P10909 & Clusterin & CLU \\
\hline P15328 & Folate receptor alpha & FOLR1 \\
\hline H3BMA1 & Mesothelin & MSLN \\
\hline P07996 & Thrombospondin-1 & THBS1 \\
\hline P98160 & Basement membrane-specific heparan sulfate proteoglycan core protein & HSPG2 \\
\hline P80188 & Neutrophil gelatinase-associated lipocalin & LCN2 \\
\hline P30530 & Tyrosine-protein kinase receptor UFO & AXL \\
\hline E2D5S3 & MHC class I antigen & HLA-C \\
\hline P05187 & Alkaline phosphatase, placental type & ALPP \\
\hline P07339 & Cathepsin D & CTSD \\
\hline P17936 & Insulin-like growth factor binding protein 3 & IGFBP3 \\
\hline D6W5P7 & ADAM metallopeptidase domain 22 & ADAM22 \\
\hline P50897 & Palmitoyl-protein thioesterase 1 & PPT1 \\
\hline Q13740 & CD166 antigen & ALCAM \\
\hline Q92820 & Gamma-glutamyl hydrolase & GGH \\
\hline P41271 & Neuroblastoma suppressor of tumorigenicity 1 & NBL1 \\
\hline B9EJB8 & Collagen, type XII, alpha 1 & COL12A1 \\
\hline P07942 & Laminin subunit beta-1 & LAMB1 \\
\hline P51884 & Lumican & LUM \\
\hline O94907 & Dickkopf-related protein 1 & DKK1 \\
\hline Q03405 & Urokinase plasminogen activator surface receptor & PLAUR \\
\hline P56199 & Integrin alpha-1 & ITGA1 \\
\hline Q9BY76 & Angiopoietin-related protein 4 & ANGPTL4 \\
\hline $\mathrm{P} 02750$ & Leucine-rich alpha-2-glycoprotein & LRG1 \\
\hline C9JBB3 & Tissue factor pathway inhibitor & TFPI \\
\hline M0QZZ9 & Mucin-16 & MUC16 \\
\hline P28799 & Granulins & GRN \\
\hline Q54A51 & Basigin (Ok blood group), isoform CRA_a & hEMMPRIN \\
\hline $\mathrm{P} 11717$ & Insulin-like growth factor 2 receptor & IGF2R \\
\hline
\end{tabular}

alterations of protein glycosylation during multi-drug resistance in cancer. In our study, we identified and quantified the differences of glycosites and site-specific glycoforms of L1CAM, TIMP1 and CLU between SGC7901 and its MDR derivates, and found significant changes on glycosites as well as site-specific glycans. This suggested that glycosylation at specific glycosites might possibly have an enormous impact on actions of the three proteins in MDR of gastric cancer. Further researches are needed to confirm this plausible conclusion.

In conclusion, our present work could well characterize the site-specific glycosylation of secreted glycoproteins, including the glycosite occupancy as well as glycan structures. And, we believe that glycosylation at specific positions and its changes in secreted glycoproteins would be greatly involved in the acquisition of MDR, and 
our work could provide new insights into the sophisticated mechanisms underlying MDR in gastric cancer. Furthermore, the aberrantly glycosylated secreted glycoproteins obtained in our work would be candidate biomarkers for MDR prediction or key targets to reverse drug resistance in gastric cancer. Further investigations are warranted to verify this.
A Tyrosine-protein kinase receptor UFO, AXL

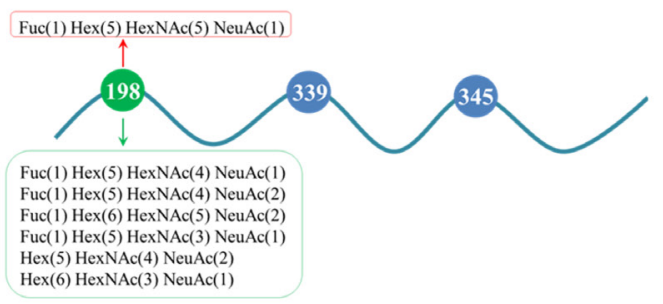

C Tissue inhibitor of metalloproteinase 1, TIMP1

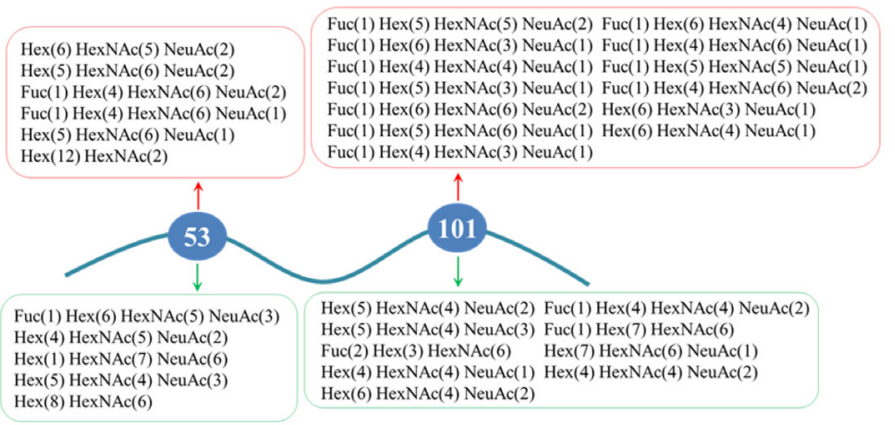

B

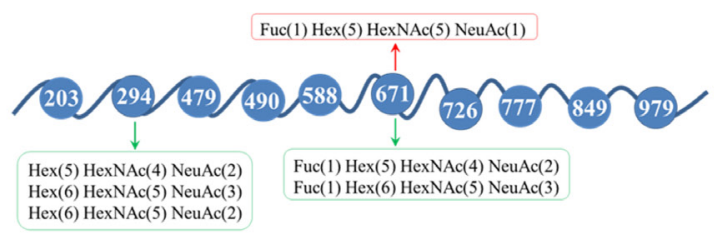

D

Clusterin, CLU

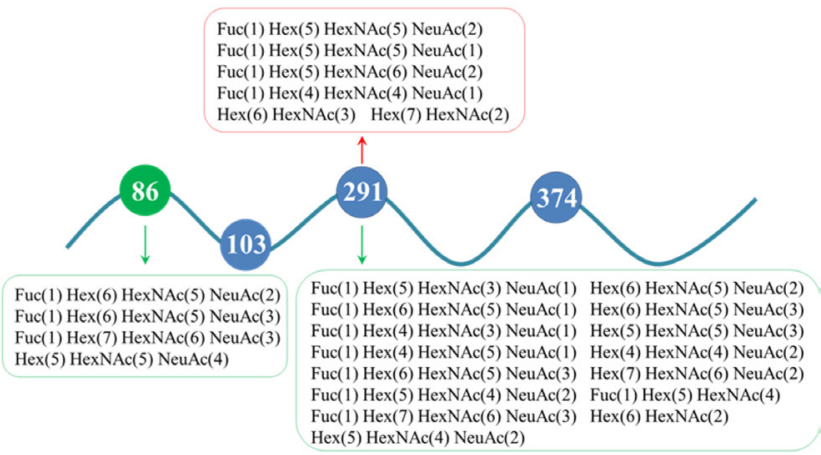

Figure 5: Schemes for changes of glycosites and site-specific N-glycosylation in MDR cells of (A) AXL; (B) L1CAM; (C) TIMP1; and (D) CLU. N-glycans that were up-regulated in MDR cells were placed above the peptides and marked red, while those down-regulated in MDR cells were put under the peptides and marked green. Besides, glycosites significantly decreased in MDR cells were marked green.

A

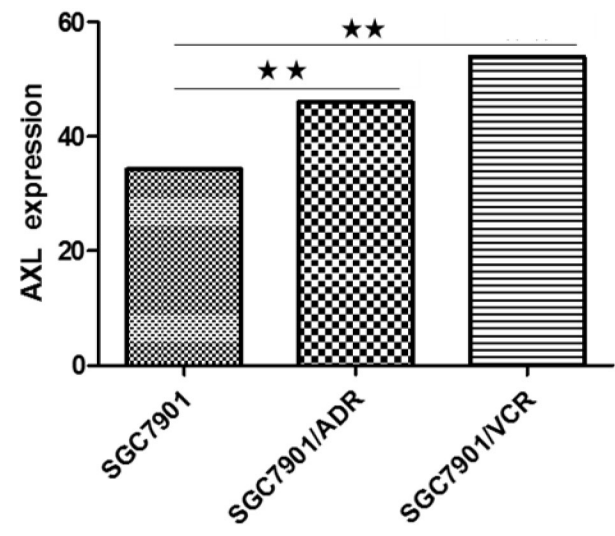

B

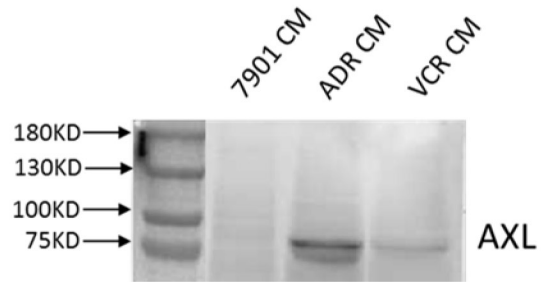

C

Kaplan-Meier Curves of AXL

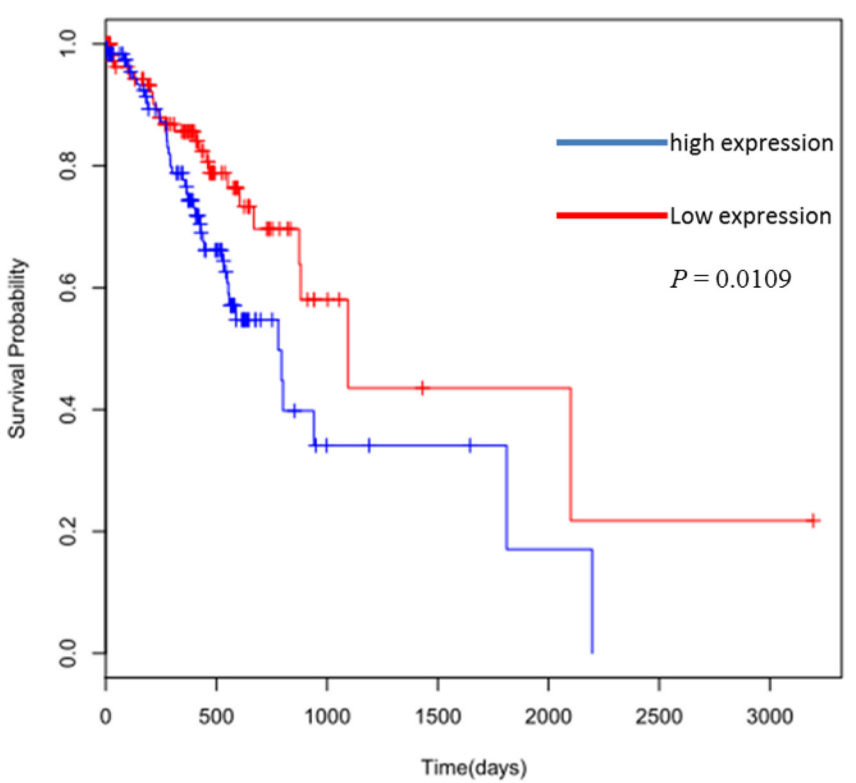

Figure 6: AXL is increased in GC MDR cells and predicts poor survival in GC patients. (A) The mRNA expression of AXL is upregulated in GC MDR cells. $P<0.01$. (B) The protein expression of AXL is increased in the conditioned medium of GC MDR cells. CM: conditioned medium. C. Based on TCGA database, high expression of tumor AXL predicts worse survival of GC patients. 


\section{MATERIALS AND METHODS}

\section{Reagents and materials}

Trypsin, 1, 4-dithiothreitol (DTT), iodacetamide (IAA), trifluoroacetic acid (TFA) and formic acid (FA) were obtained from Sigma (St. Louis, MO, USA). PNGase F was purchased from New England Biolabs (Ipswich, MA, USA). The centrifugal filter units (Amicon Ultra, $3 \mathrm{KD}, 15 \mathrm{~mL}$ ) were purchased from Millipore (Milford, MA, USA). Acetonitrile (ACN, HPLC grade) was from Merck (Darmstadt, Germany). Water used in all experiments was purified with a Milli-Q system (Millipore, Milford, MA, USA). C18 AQ beads $(3 \mu \mathrm{m}, 120 \AA$ ) were from Michrom BioResources (Auburn, CA, USA). The click maltose-HILIC beads were obtained from Xinmiao Liang group (liangxm@dicp.ac.cn).

\section{Cell culture and secretome collection}

Human gastric adenocarcinoma cell line SGC7901 was obtained from the Academy of Military Medical Science (Beijing, China). And the MDR sublines, SGC7901/VCR (VCR) and SGC7901/ADR (ADR), were developed by stepwise selection with vincristine and adriamycin, respectively. The above three cell lines were maintained in RPMI-1640 medium supplemented with $10 \%$ fetal bovine serum (FBS), $100 \mathrm{U} / \mathrm{ml}$ penicillin sodium and $100 \mathrm{mg} / \mathrm{ml}$ streptomycin sulphate at $37^{\circ} \mathrm{C}$ in a humidified atmosphere containing $5 \% \mathrm{CO}_{2}$. Vincristine $(1 \mu \mathrm{g} / \mathrm{ml})$ or adriamycin $(0.5 \mu \mathrm{g} / \mathrm{ml})$ was added to the culture medium of corresponding MDR cell sublines to maintain their MDR phenotype. The gastric cancer cell lines were appropriately cultured until $\sim 70 \%$ confluence (150 $\mathrm{mm}$ cell culture dish). In order to eliminate the possible interference of residual FBS, the cancer cells were washed with PBS (phosphate buffered saline) and serum-free RPMI-1640 medium, respectively. Then, cells were maintained in $30 \mathrm{ml}$ serum-free RPMI-1640 medium at $37^{\circ} \mathrm{C}$ for another $24 \mathrm{~h}$. The conditioned medium was collected and centrifuged at $1000 \mathrm{~g}$ to remove cell debris. Then the secreted proteins samples were concentrated using 3 KD MWCO Ultra Centrifugal Filters. The secreted protein concentration was determined by BCA assay.

\section{Protein digestion and glycopeptide enrichment}

The protein samples were prepared following the previously reported with some modifications. Briefly, 0.8 $\mathrm{mg}$ secreted proteins were reduced by DTT at $37^{\circ} \mathrm{C}$ for $2 \mathrm{~h}$ and alkylated by IAA in dark at room temperature for $30 \mathrm{~min}$. Then, trypsin was added with ratio at 1:50 (trypsin/ proteins, w/w), and the mix was incubated at $37^{\circ} \mathrm{C}$ overnight. The trypsin was again added at the same weight ratio, and incubated at $37^{\circ} \mathrm{C}$ for another $4 \mathrm{~h}$. The tryptic digests were desalted by SPE column (Waters, USA) and dried for further analysis.
The glycopeptide enrichment was performed with click maltose-HILIC materials. The prepared materials $(10 \mathrm{mg})$ were washed by $80 \% \mathrm{ACN} / 1 \% \mathrm{TFA}$, and the digestions of secreted proteins $(160 \mu \mathrm{g})$ were mixed with HILIC materials. After incubated at room temperature for $90 \mathrm{~min}$, the supernatant was removed by centrifugation at 20, $000 \mathrm{~g}$ for $3 \mathrm{~min}$, and the HILIC materials were further washed with $200 \mu \mathrm{L} 80 \%$ ACN/1\% TFA for three times. Finally, the glycopeptides were eluted by using $100 \mu \mathrm{L}$ $30 \% \mathrm{ACN} / 1 \% \mathrm{FA}$ solution for twice, and the eluted fractions were combined. The enriched glycopeptides were divided into two portions, and one part of glycopeptides was deglycosylated by using PNGase F (500 units) in $10 \mathrm{mM} \mathrm{NH}_{4} \mathrm{HCO}_{3}(\mathrm{pH} 8.0)$ at $37^{\circ} \mathrm{C}$ overnight. Then, the enriched glycopeptides and de-glycopeptides from about $50 \mu \mathrm{g}$ tryptic digestions of proteins were dried for MS analysis, respectively.

\section{Mass spectrometric analysis}

The LC-MS/MS analyses were performed on a Q-Exactive mass spectrometer (Thermo, San Jose, CA) equipped with an Ultimate 3000 system (Thermo, San Jose, CA) for separation. The LC-MS/MS system contained a C18 capillary trap column $(200 \mu \mathrm{m}$ i.d., C18 AQ beads $(5 \mu \mathrm{m}, 120 \AA))$ and a $12-\mathrm{cm} \mathrm{C} 18$ capillary analysis column $(75 \mu \mathrm{m}$ i.d., C18 AQ beads $(3 \mu \mathrm{m}, 120 \AA))$. Mobile phases A $\left(98 \% \mathrm{H}_{2} \mathrm{O} / 2 \%\right.$ acetonitrile/0.1\% FA) and $\mathrm{B}\left(80 \%\right.$ acetonitrile $\left./ 20 \% \mathrm{H}_{2} \mathrm{O} / 0.1 \% \quad \mathrm{FA}\right)$ were used to develop a gradient. For both glycopeptides and de-glycopeptides, the RP gradient was developed at $300 \mathrm{nl} / \mathrm{min}$ as follows: loading sample for $15 \mathrm{~min}$, from 4 to $45 \%$ buffer B for $135 \mathrm{~min}$, from 45 to $90 \%$ buffer B for $15 \mathrm{~min}$, from 90 to $90 \%$ buffer B for $10 \mathrm{~min}$, from 90 to $4 \%$ buffer B for $4 \mathrm{~min}$ and from 4 to $4 \%$ buffer B for $11 \mathrm{~min}$. The temperature of the ion transfer capillary was $220^{\circ} \mathrm{C}$ and the normalized collision energy was set to $25 \%$. The mass resolution was set to 70, 000 for full MS and 17, 500 for HCD MS/MS. Survey full scan MS was acquired from $\mathrm{m} / \mathrm{z} 400$ to 2,000 , and 15 most intense ions were selected for MS/MS scan. The dynamic exclusion was set as follows: repeat count, 1; duration, $30 \mathrm{~s}$; exclusion list size, 500; exclusion duration, $90 \mathrm{~s}$. All the samples, including intact glycopeptides and de-glycopeptides were analyzed for three runs.

\section{Mass spectrometry data analysis}

For glycosite analysis of secreted proteins, the mass spectrometric data of de-glycopeptides acquired by Q-Exactive MS was searched and quantified by using Maxquant (1.5.2.8) [58]. The following parameters were used for searching: mass tolerances were $20 \mathrm{ppm}$ and $0.1 \mathrm{Da}$ for the precursor and fragments, respectively; for trypsin digested samples, enzyme specificity was set to $\mathrm{KR} / \mathrm{P}$ with up to 2 missed sites; cysteine residue was set 
as a static modification of 57.0215 Da; Asn deamidation $(+0.9840 \mathrm{Da})$ and methionine oxidation (+15.9949 Da) were set as variable modifications. The glycosites were identified with the motif of N-X (Not Proline)-S/T. For the quantification of glycosites, label free quantification (LFQ) in Maxquant was employed to extract the intensity of full MS, and quantification data was further analyzed by Perseus software (version 1.5.1.6) with Log2 transform and Z-score normalization. The determination of sitespecific glycoforms was performed by using ArMone proteomics data processing platform (http://www. bioanalysis.dicp.ac.cn/proteomics/software/ArMone2. html) with some modifications [59]. First, the peptide sequence was identified using Mascot (2.3.0) following the above parameters with FDR for peptide identification of both peptides and proteins $<1 \%$. And information of $\mathrm{N}$-glycosites and peptide sequences was integrated into a format file "Peptide list" (.ppl), while the mass spectrometric data of intact glycopeptide acquired by Q-Exactive MS was converted into format of 'MZ.xml' using MS Convert. Then the '.ppl' (deglycopeptides) and 'MZ.xml' (intact glycopeptides) were loaded into Armone proteomics data processing platform. The software could automatically extract MS/MS spectra of intact glycopeptides based on oxonium ions, and the Y1 ions (peptide sequence + GlcNAc) could be determined by the characters of N-linked five core glycans. Then the molecular weight of both peptide backbone and glycans could be calculated. And the peptide back bone sequences were determined by matching the MW of Y1 ions to that from de-glycopeptides identified from Mascot search. The backbone sequences were filtered based on the maximum of $15 \mathrm{~min}$ in retention time and $20 \mathrm{ppm}$ for MW. The glycan structures were determined by using MW and fragments of specific sugars. The site-specific glycoforms could be determined by combining the two part information, and the results were further filtered with glycopeptide score more than 10 , and most of the glycan structures were further manually corrected. For the quantification of site-specific glycoforms, the intensity of glycoforms was extracted by house software, and the intensity was transformed to $\log 2$ and normalized with Z-score by using Perseus software (version 1.5.1.6). Additionally, for glycoforms quantified in the secreted proteins of three cell lines, those meeting the criteria (Ratio MDR/7901 > 2 OR < 0.5) were identified as significantly changed glycoforms. While, the glycoforms determined only in MDR cell lines (ADR\&VCR, spectra count $>2$ ) or only in SGC7901 cell line (spectra count $>5$ ) were also judged as significantly changed glycoforms.

Furthermore, the secretome was defined to proteins with the Uniprot keyword "signal or secreted" and those predicted by SignalP 4.1 [60] or SecretomeP 2.0 [61]. In addition, functional classification of the identified glycoproteins was performed by PANTHER classification system [62].

\section{TCGA data analysis}

The clinical and gene expression data of 295 primary gastric adenocarcinomas were derived from TCGA data portal (https://tcga-data.nci.nih.gov/tcga/) and TCGAbased publication [63]. The expression of AXL was divided into high and low expression group by median expression. Then, the association between AXL expression and patient survival was analyzed using the Kaplan-Meier method with the log-rank test in Cox proportional hazards model.

\section{Western blot}

The concentrated secreted protein samples collected above were resolved by $12 \%$ SDS-PAGE (Bio-Rad Laboratories, Hercules, CA, USA) and blotted onto nitrocellulose membrane (Amersham Biosciences, Pittsburgh, PA, USA). Membrane was blocked with 10\% non-fat milk at room temperature for $2 \mathrm{~h}$ and incubated overnight with primary antibody: anti-AXL (1:1000; Abcam, Cambridge, MA, USA). After three 5 min washes in TBST (Triethanolamine-Buffered Saline Solution with $0.1 \%$ Tween-20), membrane was incubated with horseradish peroxidase (HRP) conjugated secondary antibody (1:2000; Santa cruz Biotechnology, Dallas, TX, USA) for $2 \mathrm{~h}$ at room temperature and then washed again in TBST and visualized with an enhanced chemiluminescence kit (ECL-kit, santa cruz biotechnology, Dallas, TX, USA). All experiments were performed in triplicate.

\section{ACKNOWLEDGMENTS}

We would like to thank Zhe Zhang, Sijun Hu Yuanyuan Lu from Fourth Military Medical University for kind help.

\section{GRANT SUPPORT}

This work was supported by the National Key Basic Research Program of China (2014CBA02002, 2013CB911202, 2012CB910604), the Natural Science Foundation of China (No. 81430072, 81272343, $81421003,21321064,21275142,21405156,21235006)$.

\section{CONFLICTS OF INTEREST}

The authors declare no Conflicts of Interest.

\section{REFERENCES}

1. Chen W, Zheng R, Baade PD, Zhang S, Zeng H, Bray F, Jemal A, Yu XQ, He J. Cancer statistics in China, 2015. CA Cancer J Clin. 2016. 
2. Zhang D, Fan D. New insights into the mechanisms of gastric cancer multidrug resistance and future perspectives. Future Oncol. 2010; 6:527-537.

3. Lage H. ABC-transporters: implications on drug resistance from microorganisms to human cancers. Int $\mathrm{J}$ Antimicrob Agents. 2003; 22:188-199.

4. Wang F, Li T, Zhang B, Li H, Wu Q, Yang L, Nie Y, Wu K, Shi Y, Fan D. MicroRNA-19a/b regulates multidrug resistance in human gastric cancer cells by targeting PTEN. Biochem Biophys Res Commun. 2013; 434:688-694.

5. Shang Y, Zhang Z, Liu Z, Feng B, Ren G, Li K, Zhou L, Sun Y, Li M, Zhou J, An Y, Wu K, Nie Y, et al. miR-508-5p regulates multidrug resistance of gastric cancer by targeting ABCB1 and ZNRD1. Oncogene. 2014; 33:3267-3276.

6. Liu L, Sun L, Zhang H, Li Z, Ning X, Shi Y, Guo C, Han S, Wu K, Fan D. Hypoxia-mediated up-regulation of MGr1$\mathrm{Ag} / 37 \mathrm{LRP}$ in gastric cancers occurs via hypoxia-induciblefactor 1-dependent mechanism and contributes to drug resistance. Int J Cancer. 2009; 124:1707-1715.

7. Shang Y, Feng B, Zhou L, Ren G, Zhang Z, Fan X, Sun Y, Luo G, Liang J, Wu K, Nie Y, Fan D. The miR27b-CCNG1P53-miR-508-5p axis regulates multidrug resistance of gastric cancer. Oncotarget. 2015; doi: 10.18632/oncotarget.6374.

8. Wu Q, Yang Z, Xia L, Nie Y, Wu K, Shi Y, Fan D. Methylation of miR-129-5p CpG island modulates multi-drug resistance in gastric cancer by targeting $\mathrm{ABC}$ transporters. Oncotarget. 2014; 5:11552-11563; doi: 10.18632/oncotarget.2594.

9. Straussman R, Morikawa T, Shee K, Barzily-Rokni M, Qian ZR, Du J, Davis A, Mongare MM, Gould J, Frederick DT, Cooper ZA, Chapman PB, Solit DB, et al. Tumour micro-environment elicits innate resistance to RAF inhibitors through HGF secretion. Nature. 2012; 487:500-504.

10. Hirata E, Girotti MR, Viros A, Hooper S, Spencer-Dene B, Matsuda M, Larkin J, Marais R, Sahai E. Intravital imaging reveals how BRAF inhibition generates drug-tolerant microenvironments with high integrin beta1/FAK signaling. Cancer Cell. 2015; 27:574-588.

11. Junttila MR, de Sauvage FJ. Influence of tumour microenvironment heterogeneity on therapeutic response. Nature. 2013; 501:346-354.

12. Ruffell B, Coussens LM. Macrophages and therapeutic resistance in cancer. Cancer Cell. 2015; 27:462-472.

13. Obenauf AC, Zou Y, Ji AL, Vanharanta S, Shu W, Shi H, Kong X, Bosenberg MC, Wiesner T, Rosen N, Lo RS, Massague J. Therapy-induced tumour secretomes promote resistance and tumour progression. Nature. 2015; 520:368-372.

14. Zhang FF, Zhu YF, Zhao QN, Yang DT, Dong YP, Jiang L, Xing WX, Li XY, Xing H, Shi M, Chen Y, Bruce IC, Jin J, et al. Microvesicles mediate transfer of P-glycoprotein to paclitaxel-sensitive A2780 human ovarian cancer cells, conferring paclitaxel-resistance. Eur J Pharmacol. 2014; 738:83-90.

15. Choi DY, You S, Jung JH, Lee JC, Rho JK, Lee KY, Freeman MR, Kim KP, Kim J. Extracellular vesicles shed from gefitinib-resistant nonsmall cell lung cancer regulate the tumor microenvironment. Proteomics. 2014; 14: 1845-1856.

16. Xiu P, Dong X, Dong X, Xu Z, Zhu H, Liu F, Wei Z, Zhai B, Kanwar JR, Jiang H, Li J, Sun X. Secretory clusterin contributes to oxaliplatin resistance by activating Akt pathway in hepatocellular carcinoma. Cancer Sci. 2013; 104:375-382.

17. Huang H, Wang L, Li M, Wang X, Zhang L. Secreted clusterin (sCLU) regulates cell proliferation and chemosensitivity to cisplatin by modulating ERK1/2 signals in human osteosarcoma cells. World J Surg Oncol. 2014; 12:255.

18. Liu Z, Xu J, He J, Zheng Y, Li H, Lu Y, Qian J, Lin P, Weber DM, Yang J, Yi Q. A critical role of autocrine sonic hedgehog signaling in human CD138+ myeloma cell survival and drug resistance. Blood. 2014; 124:2061-2071.

19. Yuan G, Regel I, Lian F, Friedrich T, Hitkova I, Hofheinz RD, Strobel P, Langer R, Keller G, Rocken C, Zimmermann W, Schmid RM, Ebert MPA, et al. WNT6 is a novel target gene of caveolin-1 promoting chemoresistance to epirubicin in human gastric cancer cells. Oncogene. 2013; 32:375-387.

20. Cho HJ, Kim IK, Park SM, Baek KE, Nam IK, Park SH, Ryu KJ, Choi J, Ryu J, Hong SC, Jeong SH, Lee YJ, Ko GH, et al. VEGF-C mediates RhoGDI2-induced gastric cancer cell metastasis and cisplatin resistance. Int $\mathrm{J}$ Cancer. 2014; 135:1553-1563.

21. Huang H, Han Y, Wu J, Tian ZH, Qu LK, Shou CC. [Establishment of drug resistant cell line of MGC-803 and analysis of differential secretome]. Beijing Da Xue Xue Bao. 2014; 46:183-189.

22. Li K, Sun Z, Zheng J, Lu Y, Bian Y, Ye M, Wang X, Nie Y, Zou H, Fan D. In-depth research of multidrug resistance related cell surface glycoproteome in gastric cancer. J Proteomics. 2013; 82:130-140.

23. Lattova E, Tomanek B, Bartusik D, Perreault $\mathrm{H}$. $\mathrm{N}$-glycomic changes in human breast carcinoma MCF-7 and T-lymphoblastoid cells after treatment with herceptin and herceptin/Lipoplex. J Proteome Res. 2010; 9:1533-1540.

24. Lattova E, Bartusik D, Spicer V, Jellusova J, Perreault H, Tomanek B. Alterations in glycopeptides associated with herceptin treatment of human breast carcinoma mcf-7 and T-lymphoblastoid cells. Mol Cell Proteomics. 2011; 10:M111 007765.

25. Nakano M, Saldanha R, Gobel A, Kavallaris M, Packer NH. Identification of glycan structure alterations on cell membrane proteins in desoxyepothilone B resistant leukemia cells. Mol Cell Proteomics. 2011; 10:M111 009001.

26. Hoffmann M, Marx K, Reichl U, Wuhrer M, Rapp E. Sitespecific O-Glycosylation Analysis of Human Blood Plasma Proteins. Mol Cell Proteomics. 2016; 15:624-641.

27. Sun S, Shah P, Eshghi ST, Yang W, Trikannad N, Yang S, Chen L, Aiyetan P, Hoti N, Zhang Z, Chan DW, Zhang H. Comprehensive analysis of protein glycosylation by 
solid-phase extraction of N-linked glycans and glycositecontaining peptides. Nat Biotechnol. 2016; 34:84-88.

28. Shah P, Wang X, Yang W, Toghi Eshghi S, Sun S, Hoti N, Chen L, Yang S, Pasay J, Rubin A, Zhang H. Integrated Proteomic and Glycoproteomic Analyses of Prostate Cancer Cells Reveal Glycoprotein Alteration in Protein Abundance and Glycosylation. Mol Cell Proteomics. 2015; 14:2753-2763.

29. Cheng K, Chen R, Seebun D, Ye M, Figeys D, Zou H. Large-scale characterization of intact $\mathrm{N}$-glycopeptides using an automated glycoproteomic method. J Proteomics. 2014; 110:145-154.

30. Toricelli M, Melo FH, Peres GB, Silva DC, Jasiulionis MG. Timp1 interacts with beta-1 integrin and CD63 along melanoma genesis and confers anoikis resistance by activating PI3-K signaling pathway independently of Akt phosphorylation. Mol Cancer. 2013; 12:22.

31. Ma H, Miao X, Ma Q, Zheng W, Zhou H, Jia L. Functional roles of glycogene and $\mathrm{N}$-glycan in multidrug resistance of human breast cancer cells. IUBMB Life. 2013; 65:409-422.

32. Guo H, Dong J, Hu S, Cai X, Tang G, Dou J, Tian M, He F, Nie Y, Fan D. Biased random walk model for the prioritization of drug resistance associated proteins. Sci Rep. 2015; 5:10857.

33. Chen Z, Zhang L, Xia L, Jin Y, Wu Q, Guo H, Shang X, Dou J, Wu K, Nie Y, Fan D. Genomic analysis of drug resistant gastric cancer cell lines by combining mRNA and microRNA expression profiling. Cancer Lett. 2014; 350:43-51.

34. Cheng L, Luo S, Jin C, Ma H, Zhou H, Jia L. FUT family mediates the multidrug resistance of human hepatocellular carcinoma via the PI3K/Akt signaling pathway. Cell Death Dis. 2013; 4:e923.

35. Paccez JD, Vogelsang M, Parker MI, Zerbini LF. The receptor tyrosine kinase Axl in cancer: biological functions and therapeutic implications. Int J Cancer. 2014; 134:1024-1033.

36. Graham DK, DeRyckere D, Davies KD, Earp HS. The TAM family: phosphatidylserine sensing receptor tyrosine kinases gone awry in cancer. Nat Rev Cancer. 2014; 14:769-785.

37. Zhang Z, Lee JC, Lin L, Olivas V, Au V, LaFramboise T, Abdel-Rahman M, Wang X, Levine AD, Rho JK, Choi YJ, Choi CM, Kim SW, et al. Activation of the AXL kinase causes resistance to EGFR-targeted therapy in lung cancer. Nat Genet. 2012; 44:852-860.

38. Li Y, Jia L, Liu C, Gong Y, Ren D, Wang N, Zhang X, Zhao Y. Axl as a downstream effector of TGF-betal via PI3K/Akt-PAK1 signaling pathway promotes tumor invasion and chemoresistance in breast carcinoma. Tumour Biol. 2015; 36:1115-1127.

39. Asiedu MK, Beauchamp-Perez FD, Ingle JN, Behrens MD, Radisky DC, Knutson KL. AXL induces epithelial-tomesenchymal transition and regulates the function of breast cancer stem cells. Oncogene. 2014; 33:1316-1324.

40. Elkabets M, Pazarentzos E, Juric D, Sheng Q, Pelossof RA, Brook S, Benzaken AO, Rodon J, Morse N, Yan JJ, Liu M, Das R, Chen Y, et al. AXL mediates resistance to PI3Kalpha inhibition by activating the EGFR/PKC/mTOR axis in head and neck and esophageal squamous cell carcinomas. Cancer Cell. 2015; 27:533-546.

41. Wang Y, Xia H, Zhuang Z, Miao L, Chen X, Cai H. Axlaltered microRNAs regulate tumorigenicity and gefitinib resistance in lung cancer. Cell Death Dis. 2014; 5:e1227.

42. Li J, Jia L, Ma ZH, Ma QH, Yang XH, Zhao YF. Axl glycosylation mediates tumor cell proliferation, invasion and lymphatic metastasis in murine hepatocellular carcinoma. World J Gastroenterol. 2012; 18:5369-5376.

43. Ren D, Li Y, Gong Y, Xu J, Miao X, Li X, Liu C, Jia L, Zhao Y. Phyllodes tumor of the breast: role of Axl and ST6GalNAcII in the development of mammary phyllodes tumors. Tumour Biol. 2014; 35:9603-9612.

44. O’Bryan JP, Fridell YW, Koski R, Varnum B, Liu ET. The transforming receptor tyrosine kinase, Axl, is posttranslationally regulated by proteolytic cleavage. J Biol Chem. 1995; 270:551-557.

45. Reichl P, Fang M, Starlinger P, Staufer K, Nenutil R, Muller P, Greplova K, Valik D, Dooley S, Brostjan C, Gruenberger T, Shen J, Man K, et al. Multicenter analysis of soluble Axl reveals diagnostic value for very early stage hepatocellular carcinoma. Int J Cancer. 2015; 137:385-394.

46. Gustafsson A, Martuszewska D, Johansson M, Ekman C, Hafizi S, Ljungberg B, Dahlback B. Differential expression of Axl and Gas6 in renal cell carcinoma reflecting tumor advancement and survival. Clin Cancer Res. 2009; 15:4742-4749.

47. Yoon H, Min JK, Lee DG, Kim DG, Koh SS, Hong HJ. L1 cell adhesion molecule and epidermal growth factor receptor activation confer cisplatin resistance in intrahepatic cholangiocarcinoma cells. Cancer Lett. 2012; 316:70-76.

48. Kiefel H, Bondong S, Erbe-Hoffmann N, Hazin J, Riedle S, Wolf J, Pfeifer M, Arlt A, Schafer H, Muerkoster SS, Altevogt P. L1CAM-integrin interaction induces constitutive NF-kappaB activation in pancreatic adenocarcinoma cells by enhancing IL-1beta expression. Oncogene. 2010; 29:4766-4778.

49. Sebens Muerkoster S, Werbing V, Sipos B, Debus MA, Witt M, Grossmann M, Leisner D, Kotteritzsch J, Kappes H, Kloppel G, Altevogt P, Folsch UR, Schafer H. Drug-induced expression of the cellular adhesion molecule L1CAM confers anti-apoptotic protection and chemoresistance in pancreatic ductal adenocarcinoma cells. Oncogene. 2007; 26:2759-2768.

50. Bjerre $\mathrm{C}$, Vinther L, Belling KC, Wurtz SO, Yadav R, Lademann U, Rigina O, Do KN, Ditzel HJ, Lykkesfeldt AE, Wang J, Nielsen HB, Brunner N, et al. TIMP1 overexpression mediates resistance of MCF7 human breast cancer cells to fulvestrant and downregulates progesterone receptor expression. Tumour Biol. 2013; 34:3839-3851.

51. Song T, Dou C, Jia Y, Tu K, Zheng X. TIMP-1 activated carcinoma-associated fibroblasts inhibit tumor apoptosis by activating SDF1/CXCR4 signaling in hepatocellular 
carcinoma. Oncotarget. 2015; 6:12061-12079. doi: 10.18632/oncotarget.3616.

52. Schrohl AS, Meijer-van Gelder ME, Holten-Andersen MN, Christensen IJ, Look MP, Mouridsen HT, Brunner N, Foekens JA. Primary tumor levels of tissue inhibitor of metalloproteinases-1 are predictive of resistance to chemotherapy in patients with metastatic breast cancer. Clin Cancer Res. 2006; 12:7054-7058.

53. Zhang H, Kim JK, Edwards CA, Xu Z, Taichman R, Wang CY. Clusterin inhibits apoptosis by interacting with activated Bax. Nat Cell Biol. 2005; 7:909-915.

54. Park DC, Yeo SG, Wilson MR, Yerbury JJ, Kwong J, Welch WR, Choi YK, Birrer MJ, Mok SC, Wong KK. Clusterin interacts with Paclitaxel and confer Paclitaxel resistance in ovarian cancer. Neoplasia. 2008; 10:964-972.

55. Kim YS, Ahn YH, Song KJ, Kang JG, Lee JH, Jeon SK, Kim HC, Yoo JS, Ko JH. Overexpression and beta-1, 6-N-acetylglucosaminylation-initiated aberrant glycosylation of TIMP-1: a "double whammy" strategy in colon cancer progression. J Biol Chem. 2012; 287:32467-32478.

56. Kim H, Saldova R, Park JH, Lee YH, Harvey DJ, Wormald MR, Wynne K, Elia G, Kim HJ, Rudd PM, Lee ST. The presence of outer arm fucose residues on the N-glycans of tissue inhibitor of metalloproteinases-1 reduces its activity. J Proteome Res. 2013; 12:3547-3560.
57. Rohne P, Prochnow H, Wolf S, Renner B, Koch-Brandt C. The chaperone activity of clusterin is dependent on glycosylation and redox environment. Cell Physiol Biochem. 2014; 34:1626-1639.

58. Cox J, Hein MY, Luber CA, Paron I, Nagaraj N, Mann M. Accurate proteome-wide label-free quantification by delayed normalization and maximal peptide ratio extraction, termed MaxLFQ. Mol Cell Proteomics. 2014; 13:2513-2526.

59. Jiang X, Ye M, Cheng K, Zou H. ArMone: a software suite specially designed for processing and analysis of phosphoproteome data. J Proteome Res. 2010; 9:2743-2751.

60. Petersen TN, Brunak S, von Heijne G, Nielsen H. SignalP 4.0: discriminating signal peptides from transmembrane regions. Nat Methods. 2011; 8:785-786.

61. Bendtsen JD, Jensen LJ, Blom N, Von Heijne G, Brunak S. Feature-based prediction of non-classical and leaderless protein secretion. Protein Eng Des Sel. 2004; 17:349-356.

62. Mi H, Muruganujan A, Casagrande JT, Thomas PD. Large-scale gene function analysis with the PANTHER classification system. Nat Protoc. 2013; 8:1551-1566.

63. Cancer Genome Atlas Research N. Comprehensive molecular characterization of gastric adenocarcinoma. Nature. 2014; 513:202-209. 